\title{
Recurso Educacional Aberto para Treinamento Inicial e Contínuo em Mamografia Utilizando Questionário Virtual e Técnicas de Gamificação
}

\author{
Paulo C. Silva ${ }^{1}$, Gabriel F. Cyrino ${ }^{2}$, Edgard Lamounier ${ }^{1,2}$, \\ Alexandre Cardoso ${ }^{1,2}$, Ana C. Patrocinio ${ }^{1}$ \\ ${ }^{1}$ Programa de Pós-graduação em Engenharia Biomédica \\ Faculdade de Engenharia Elétrica (FEELT) \\ Universidade Federal de Uberlândia (UFU) - Uberlândia - MG - Brasil \\ ${ }^{2}$ Programa de Pós-graduação em Engenharia Elétrica \\ Faculdade de Engenharia Elétrica (FEELT) \\ Universidade Federal de Uberlândia (UFU) - Uberlândia - MG - Brasil \\ \{paulo.camargos, gabrielcyrino, lamounier, alexandre, ana.patrocinio\}@ufu.br
}

\begin{abstract}
The training of students and technicians in mammography is essential for high quality radiological images, with a direct impact on the ability to diagnose breast cancer. Observing the gap in the initial and continuous training of professionals in this area for several reasons, this work presents an open educational resource for training in mammography. This work presents the MEP (Mammography Education Platform) system, which uses gamification techniques through a virtual environment associated with a questionnaire and management module. The created system has a potential for use in initial courses and continuous training, as well as in quality control programs.
\end{abstract}

Resumo. O treinamento de estudantes e técnicos em mamografia é fundamental para uma alta qualidade de imagens radiológicas, com impacto direto na capacidade de diagnóstico do câncer de mama. Observando a defasagem no treinamento inicial e contínuo dos profissionais desta área por motivos diversos, este trabalho apresenta um recurso educacional aberto para treinamento em mamografia. Este trabalho apresenta o sistema MEP (Mammography Education Platform), que utiliza técnicas de gamificação através de um ambiente virtual associado a um módulo de questionários e gerenciamento. O sistema criado possui um potencial de utilização em cursos iniciais e de treinamento contínuo, tal como em programas de controle de qualidade.

\section{Introdução}

O câncer de mama é uma grande preocupação na saúde feminina e masculina. Este é o segundo tipo mais comum de neoplasma maligno, logo após o câncer de pulmão. No Brasil, entre as mulheres, é o principal câncer diagnosticado (sem considerar o câncer de pele não-melanoma) e a principal causa de morte [Bray et al. 2018].

Foi estimado um total de 66.280 casos por ano em um levantamento do INCA (Instituto Nacional de Câncer) para o triênio 2020-2022, 
totalizando $29,7 \%$ de todas as categorias de câncer entre as mulheres [INCA - Instituto Nacional de Câncer José Alencar Gomes da Silva 2020].

A maioria dos casos são diagnosticados através da mamografia. Ela é considerada um padrão internacional para o rastreamento e diagnóstico de câncer de mama [Coop et al. 2016]. Este é um exame de rotina para mulheres com idade entre 50 e 69 anos, sendo a sua realização recomendada a cada 2 anos segundo o Ministério da Saúde do Brasil.

A mamografia é um exame que utiliza raios $X$ para geração de imagens que possibilitam a visualização de estruturas internas da mama, bem como estruturas anômalas (calcificações e nódulos). Para a realização destes exames, é necessário um(a) técnico(a) em radiologia qualificado(a) para operação do equipamento de imagem.

A acurácia do diagnóstico está relacionada à qualidade da imagem, que depende da aquisição de imagem adequada, das condições do equipamento mamográfico e expertise do radiologista. Isto é, técnicas como posicionamento correto e aplicação da compressão correta na mama afetam diretamente as características das imagens.

Em um estudo [Sabino et al. 2014] que abordou implementação de um programa de controle de qualidade, foi mostrado que $89 \%$ das falhas nos exames foram associadas ao mau-posicionamento das mamas. Este estudo destacou a necessidade de treinamento contínuo dos profissionais.

Além disso, a forma como o exame é realizado também afeta a taxa de recall (retorno) de pacientes. Em outro estudo [Agius and Naylor 2018], metade dos pacientes relataram dor moderada no momento da compressão da mama. Isto resulta em desencorajamento de outras pessoas a realizarem o exame, atrasos e não-comparecimento a consultas marcadas [Agius and Naylor 2018]. Ademais, exames com falhas geram a necessidade de recall para reexecução do mesmo. Isto submete pacientes à exposição a doses maiores de radiação e as desestimulam a realizarem os os exames de rotina e rastreamento do câncer de mama.

O estudo de [Sabino et al. 2014] ainda mostrou que as clínicas de mamografia mais distantes das sedes e centros de treinamento sofrem três vezes mais com falhas nos exames. Também é destacado a necessidade de acompanhamento mensal com supervisores técnicos.

Os treinamentos de profissionais em mamografia são geralmente feitos por cursos de reciclagem e/ou implementação de programas de controle de qualidade. Nestes programas de qualidade, ações são realizadas para identificação e correção de falhas por parte dos técnicos. No programa demonstrado por [Sabino et al. 2014], técnicos foram submetidos a um treinamento de $15 \mathrm{~h}$ com supervisores de radioproteção.

Com o crescimento das técnicas de computação e consequente melhoramento das técnicas em Realidade Virtual (RV), novas formas de treinamento surgiram em diversas áreas. Essas novas formas de treinamento têm mostrado grande utilidade, principalmente na área da saúde [Bigdeli and Kaufman 2017, Wang et al. 2016, Malhotra et al. 2017]. A citar, por exemplo, técnicas aplicando conceitos de gamificação e jogos sérios [Dörner et al. 2016].

Jogos sérios são semelhantes aos jogos de entretenimento, porém pode ser uti- 
lizados com um viés voltado para a educação, treinamento e tratamentos médicos [Dörner et al. 2016, De Gloria et al. 2014].

$\mathrm{Na}$ área de auxílio de tratamentos médicos/fisioterápicos, [Cyrino et al. 2018] desenvolveu um jogo sério customizável baseado em RV para auxiliar o processo de reabilitação de pessoas que sofreram derrame. O sistema conta com um painel de controle para customização do jogo e gerenciamento de pacientes. Testes foram feitos com pacientes e avaliados com questionários. Os autores do trabalho concluíram que o sistema tem um potencial de utilização em pessoas com paralisia nos membros superiores em decorrência de um derrame.

Em outro trabalho que utiliza RV, um grupo [Bridge et al. 2014] desenvolveu uma aplicação para que estudantes de imagens médicas praticassem diversas técnicas radiográficas de forma virtual, em adição a prática real, em laboratório. Uma comparação randomizada foi realizada entre um grupo de estudantes que praticou somente em laboratório (real) e outro grupo que em adição, praticou com a aplicação virtual. Foi concluído que o grupo que praticou também no ambiente virtual teve um resultado de notas em exame melhor do que o outro grupo que praticou somente em laboratório.

No trabalho de [O'Connor et al. 2021] foi desenvolvido um sistema em RV para o treinamento inicial de estudantes do primeiro ano do curso de Tecnologia em Radiologia. O sistema apresentado no trabalho utiliza HTC Vive Pro ${ }^{\mathrm{TM}}$. O sistema de treinamento era dividido em dois módulos: um modulo de questionário e outro de simulação.

Após responderem um questionário contendo questões sobre anatomia, posicionamento e patologias, um ambiente simulado contendo um paciente virtual (avatar) era apresentado. Neste ambiente simulado, os estudantes podiam manipular o equipamento radiográfico e tirar imagens simuladas de raios X. Na avaliação dos autores, os estudantes se sentiram mais confiantes na aplicação das técnicas no mundo real e apreciaram a interatividade do simulador.

Em outro estudo [Sapkaroski et al. 2018], com um sistema em RV voltado para estudantes de Tecnologia em Radiologia, autores criaram um simulador para geração de imagens de raios $\mathrm{X}$ e interação com o paciente. No sistema, os estudantes deveriam posicionar a mão de um paciente e gerar uma imagem radiográfica virtual. A simulação foi feita com o auxílio de um Oculus Rift ${ }^{\mathrm{TM}}$ ou HTC Vive ${ }^{\mathrm{TM}}$. Com este sistema, foi possível executar a comunicação do usuário com o avatar do paciente no jogo, bem como realizar vários tipos de exames, como de imagens da tórax, abdome, entre outros. O sistema foi adotado formalmente na grade curricular do curso de Bacharelado em Radiografia e Imagens Médicas, na Universidade de Monash - Austrália.

Considerando a questão de falta de treinamento contínuo em centros de mamografia distantes de suas sedes, e dado o contexto atual de isolamento social, devido à pandemia de COVID-19, o treinamento de modalidade remota surge como uma possível forma de mitigação do problema e auxílio para mudança deste cenário. Este trabalho visa propor um sistema customizável que possa ser utilizado de forma remota e de fácil acesso por estudantes e profissionais da área da mamografia.

Neste trabalho, é apresentado o MEP (Mammography Education Platform), um recurso educacional aberto para treinamento inicial e contínuo de técnicos e estudantes em mamografia utilizando técnicas de RV associadas a questionários de avaliação de co- 
nhecimento customizáveis. Assim, as técnicas utilizadas para a construção dos módulos do sistema e suas características serão demonstradas.

\section{Metodologia}

Nesta seção, serão apresentadas as ferramentas, técnicas e arquitetura utilizada na construção deste sistema.

A criação do MEP foi divida em dois segmentos principais: módulo gamificado e o módulo de questionários e gerenciamento. Esta forma de divisão do sistema de treinamento também é apresentado por [O'Connor et al. 2021].

No módulo gamificado do MEP, encontra-se um ambiente virtual em que usuários podem praticar procedimentos relacionados a mamografia, bem como receber instruções específicas de equipamentos. A especificação e descrição deste módulo é apresentado na Seção 2.1 .

No módulo de gerenciamento e questionário do MEP, compreende a parte de cadastro de usuários, questões e testes, bem como um sistema de estatísticas sobre o desempenho dos treinandos (trainees). Este módulo possui perfis de acesso diferentes e será descrito na Seção 2.2.

\subsection{Módulo Gamificado}

Esta seção descreve o processo de produção dos elementos mais importantes do cenário construído no módulo gamificado: o mamógrafo e o phantom mamográfico virtual.

Os assets utilizados no jogo foram modelados e texturizados, utilizando a suite de criação aberta Blender (v2.8). Os modelos 3D foram criados utilizando a técnica de modelagem box modeling. Para texturização foi utilizado o PBR (Physically-Based Rendering) como método, que possibilita a conservação do nível de realismo na exportação.

Todos os modelos 3D criados foram baseados em elementos existentes. Um mamógrafo (Figura 1) foi modelado com base no equipamento Siemens Mammomat ${ }^{\odot}$. $\mathrm{O}$ modelo foi escolhido devido à facilidade de acesso ao mesmo em um hospital local, que permitiu que imagens fotográficas fossem realizadas como referência para o processo de modelagem.

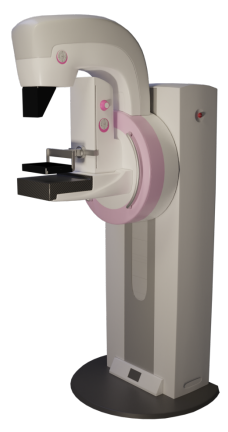

(a)

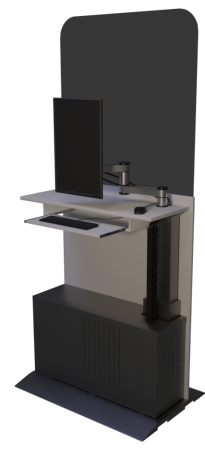

(b)

Figura 1. Renderização em perspectiva do (a) do mamógrafo e da (b) estação de comando. 
Um modelo 3D (Figura 2) de phantom mamográfico (utilizado para testes em equipamentos de mamografia) foi produzido com base no modelo $C I R S^{\oplus} 11 a$. Este modelo foi adicionado ao jogo para simular as estruturas da mama na imagem virtual de raios $\mathrm{X}$ e permitir o ensinamento de posicionamento no mamógrafo.

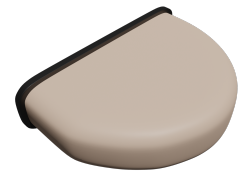

(a)

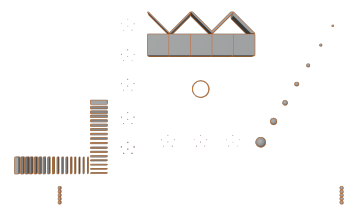

(b) Figura 2. Renderização em perspectiva do (a) phantom virtual modelado e vista
superior das (b) estruturas internas.

As cenas das fases do jogo foram criadas utilizando o programa Unity3D Engine, utilizando a pipeline de renderização HDRP (High Definition Render Pipeline). Este método de criação permite a renderização com gráficos com alto realismo nas cenas.

O jogo foi dividido em três níveis principais, cada um com um conjunto de tarefas, mais um modo livre de navegação. Este modo livre permite que o usuário interaja e navegue no ambiente sem objetivos a serem cumpridos. Em cada nível o usuário deve seguir uma sequência de instruções pré-determinadas relacionadas à operação do sistema mamográfico. Por exemplo, no primeiro nível, os usuários devem se habituar na cena, encontrar e identificar ferramentas.

Para cada operação bem-sucedida os usuários são recompensados com uma moeda virtual, denominada ROT (em homenagem ao físico Wilhelm C. Röntgen). Este mecanismo de recompensas é um dos conceitos utilizados em sistemas gamificados [Dörner et al. 2016]. As moedas podem ser eventualmente trocadas por melhorias no equipamento e na sala virtual.

Para produção dos mapas de textura dos modelos 3D, foi necessário criar um plugin para o Blender. Este plugin é responsável por converter as texturas nativas do programa em texturas aceitas pelo método HDRP no programa de criação de cenas (Unity3D).

Em uma das tarefas no jogo, o usuário deve realizar a configuração e geração de imagens mamográficas. Para isso, foi criado um script na linguagem de programação Python, para geração de imagens simuladas de raios X.

O script é executado temporariamente após acionamento do botão de execução do mamógrafo pelo usuário na cena. Este script é responsável por montar uma cena interna no Blender, configurar parâmetros internos e realizar o processo de renderização. Foi implementado um segundo script (executado como processo de fundo), responsável pela comunicação através do protocolo MQTT (Message Queuing Telemetry Transport) permitindo a intermediação do processo de renderização (script) com o jogo. A sequência de execução é demonstrada na Figura 3.

O jogo foi desenvolvido para computadores (desktops e/ou notebooks). Assim, 


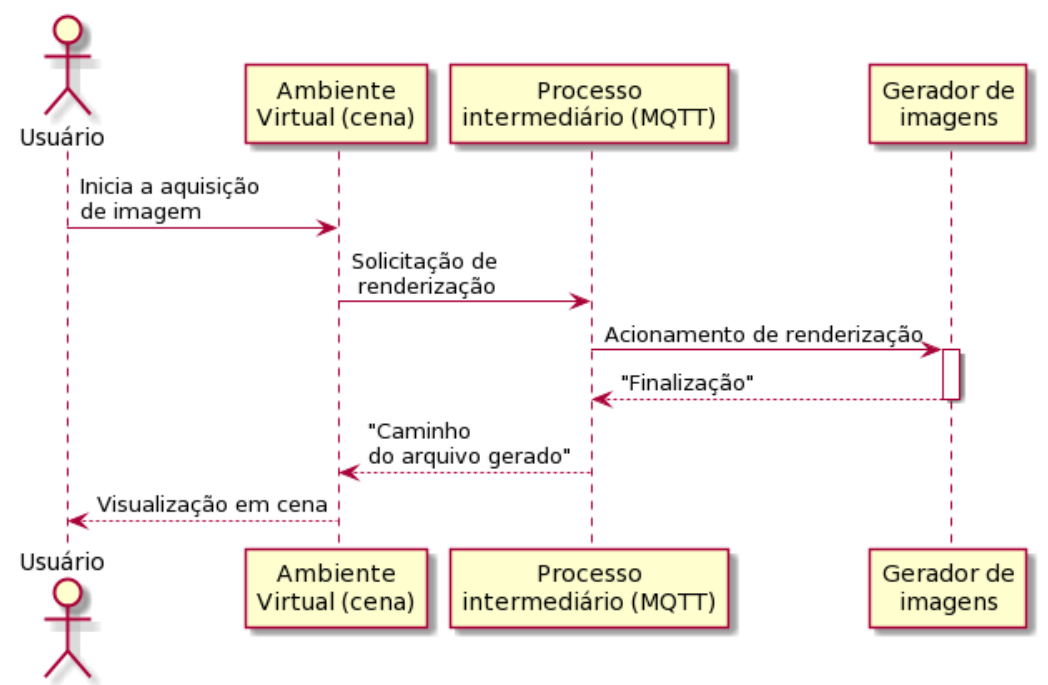

Figura 3. Diagrama de sequência demonstrando o processo de geração de imagens virtuais.

usuários podem interagir com os elementos utilizando o mouse e teclado do computador. É permitido a navegação, seleção e acionamentos utilizando esses dispositivos.

A medida que o usuário navega no ambiente, instruções contextuais são exibidas na tela do computador. Por exemplo, um painel de instruções informando ao usuário as teclas necessárias para ativar um dispositivo ou selecionar o pedal do mamógrafo.

\subsection{Módulo de Questionários e Gerenciamento}

O sistema conta com três perfis de uso: administrador, supervisor e trainee. O administrador possui total acesso às informações dos supervisores e trainees, sendo possível também o cadastro de supervisores e trainees.

O módulo de questionários e gerenciamento do MEP permite que supervisores cadastrem usuários, exames, questões e realize atribuições de tarefas aos treinandos (trainees). Do lado dos trainees, o módulo permite o acesso às tarefas atribuídas pelo supervisor responsável.

Este módulo foi desenvolvido como uma aplicação web. Foi utilizado a arquitetura REST (Representational state transfer), que facilita a comunicação entre o módulo de gamificação e o módulo gerenciamento e questionários. Assim, foi utilizado uma separação entre backend e frontend.

O backend é responsável pelas regras de negócio, bem como a comunicação com o banco de dados. Foi utilizado o Python como linguagem de programação, bem como os frameworks Django e Django Rest Framework para o desenvolvimento do backend. O frontend é responsável pela parte de interface de uso, como telas de cadastro, listas e formulários. Foram utilizadas as linguagens JavaScript, HTML (HyperText Markup Language) e CSS (Cascading Style Sheets). No frontend, também foi utilizado o framework Vue.js. O sistema de gerenciamento de banco de dados utilizado foi o PostgreSQL.

Para desenvolvimento do sistema foi utilizado a plataforma de virtualização Docker, que permite a entrega do sistema em forma de pacotes (contêineres). Isto é, a 
unificação do backend, frontend e banco de dados é realizada através desse sistema de virtualização. Isto permite a distribuição (deploy) facilitada do módulo de questionários e gerenciamento.

Os módulos de gamificação e gerenciamento são integrados de forma manual, isto é, um arquivo em formato JSON (JavaScript Object Notation) é utilizado para compartilhamento de informações. Dados sobre o jogo $(\log s)$, como a utilização do usuário no jogo, podem ser carregados pelo usuário em sua conta no módulo de questionários (web). A Figura 4 exibe uma representação das macro-interações possíveis no sistema MEP.

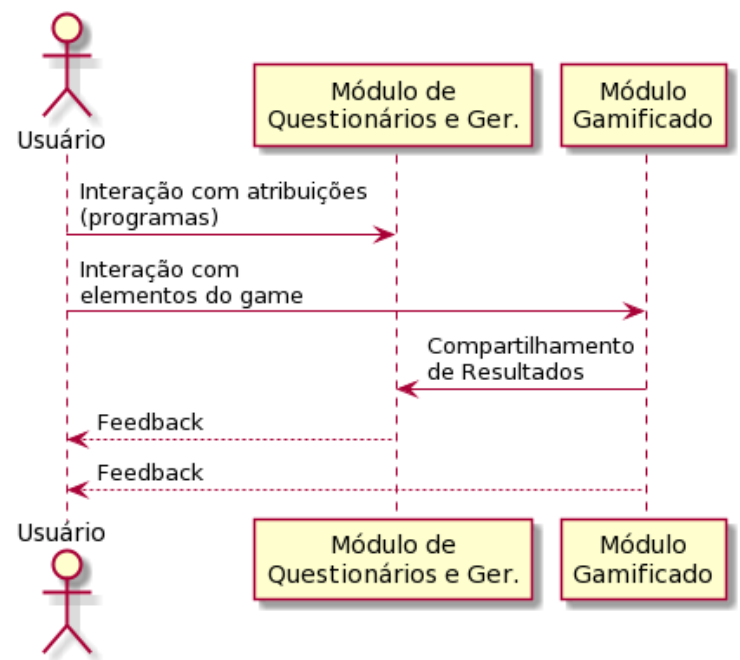

Figura 4. Diagrama representando as interações do usuário trainee, módulo gamificado e módulo de questionários e gerenciamento.

\section{Resultados}

\subsection{Modulo Gamificado}

O módulo gamificado final criado é apresentado na Figura 5. É possível observar na imagem as instruções de objetivo (topo), as instruções de teclas para operação de um determinado dispositivo (à esquerda), o dispositivo atualmente selecionado (canto superior direito) e a quantidade de recompensas (ROT) adquirida (canto superior esquerdo).

Os usuários podem navegar pelo ambiente em primeira pessoa (first person character) e podem observar detalhes dos equipamentos. Como os modelos 3D no ambiente foram modelados com base em equipamentos e dispositivos reais, os usuários recebem instruções baseado no equipamento real. Por exemplo, para posicionar a altura do arco do mamógrafo, o usuário deve selecionar o pedal e apertar a tecla em seu teclado correspondente ao botão do pedal real.

Como parte da gamificação, as recompensas adquiridas podem ser trocadas por melhorias no ambiente, como um novo pedal ou placa de compressão. No mundo real, estes elementos são vendidos pelos fabricantes de forma separada também. Este sistema de troca é frequentemente visto em jogos do tipo RPG (role-playing games).

As fases criadas no jogo contemplam elementos como alteração de parâmetros na estação de trabalho, posicionamento (rotação, altura, etc.), ativação do equipamento de 


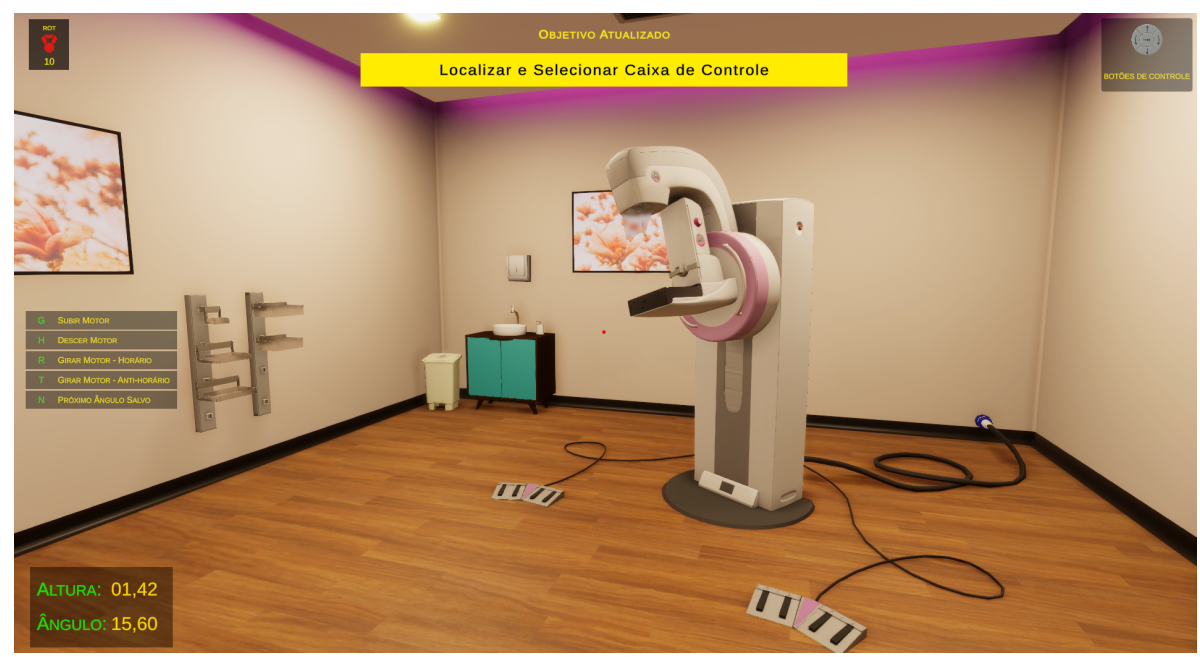

Figura 5. Captura de tela do ambiente virtual no módulo gamificado.

mamografia, seleção correta das placas de compressão, entre outros. A Figura 6 exibe uma tela com as instruções de um dispositivo.

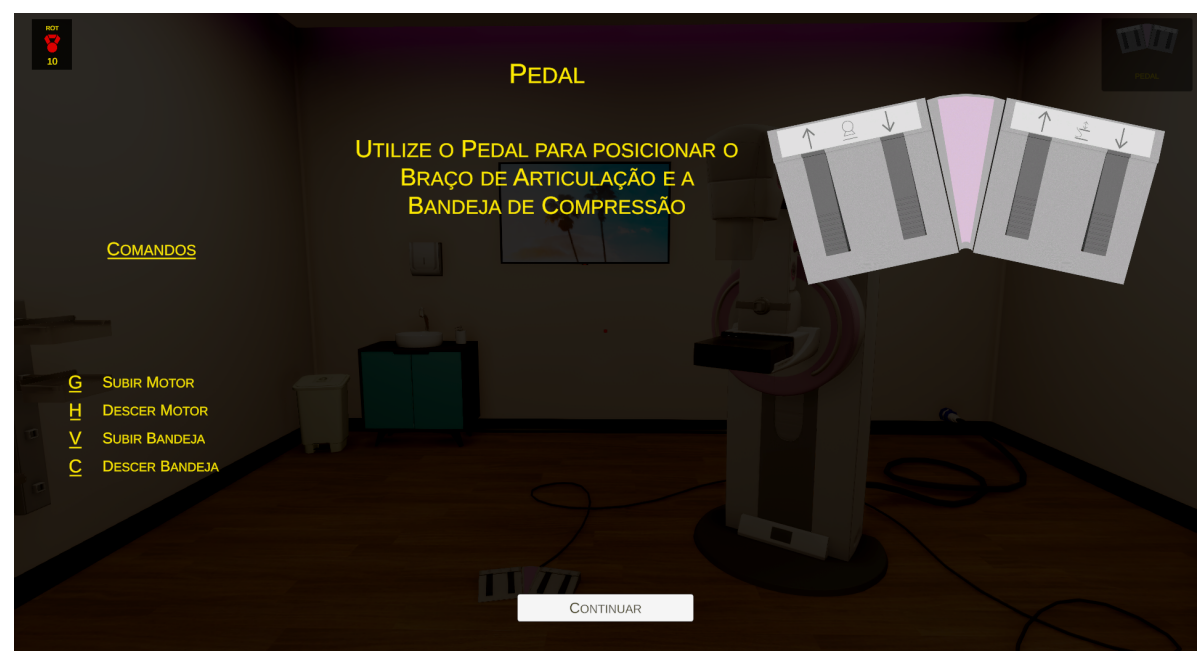

Figura 6. Tela do jogo exibindo as instruções de um dispositivo utilizado na operação do mamógrafo.

Vale destacar que a arquitetura em que o jogo foi construído permite a inserção de novas fases, com novos objetivos de uma maneira relativamente fácil. Isto é, o sistema de classes e componentes adotado no Unity $3 D$ permite a herança e override de métodos e atributos, estabelecendo a constituição de novos objetivos e instruções para as fases.

\subsection{Módulo Web de Questionários e Gerenciamento}

As Figuras 7 e 8 exibem as telas do módulo web de questionários e gerenciamento desenvolvido. O acesso é realizado através de um dos três perfis criados (Seção 2.2).

O módulo permite que um supervisor cadastre um trainee e crie programas de treinamento. Um programa de treinamento é constituído por uma ou mais solicitações de exames (questionários) e pela solicitação de execução do módulo gamificado (opcional). Desta forma, os programas devem ser atribuídos aos trainees. 


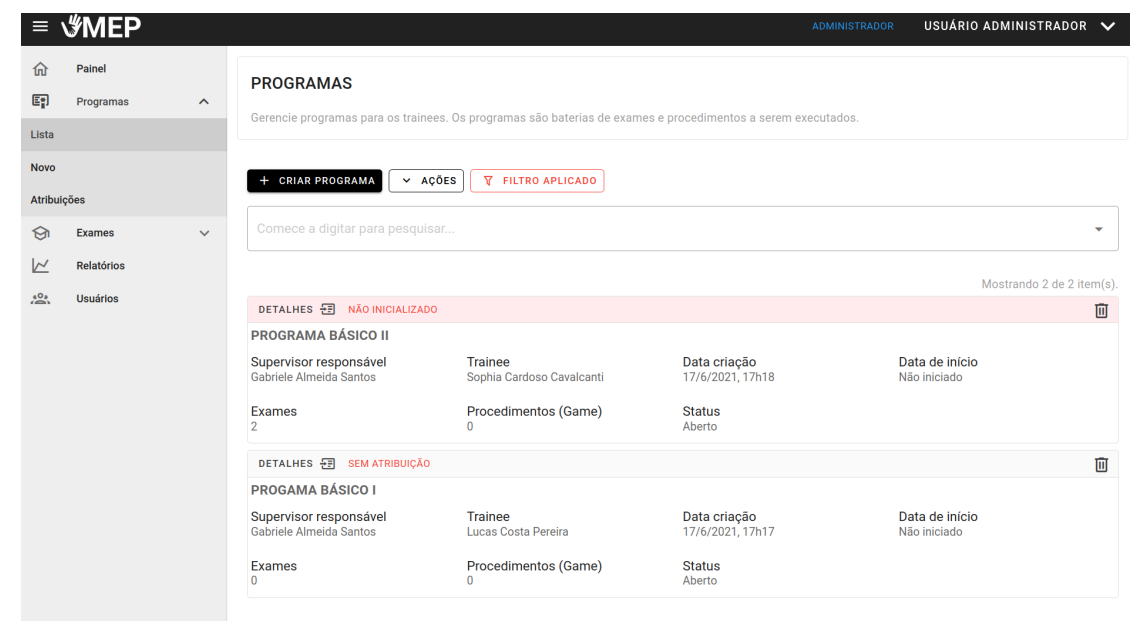

Figura 7. Captura de tela mostrando a lista de programas cadastrados.

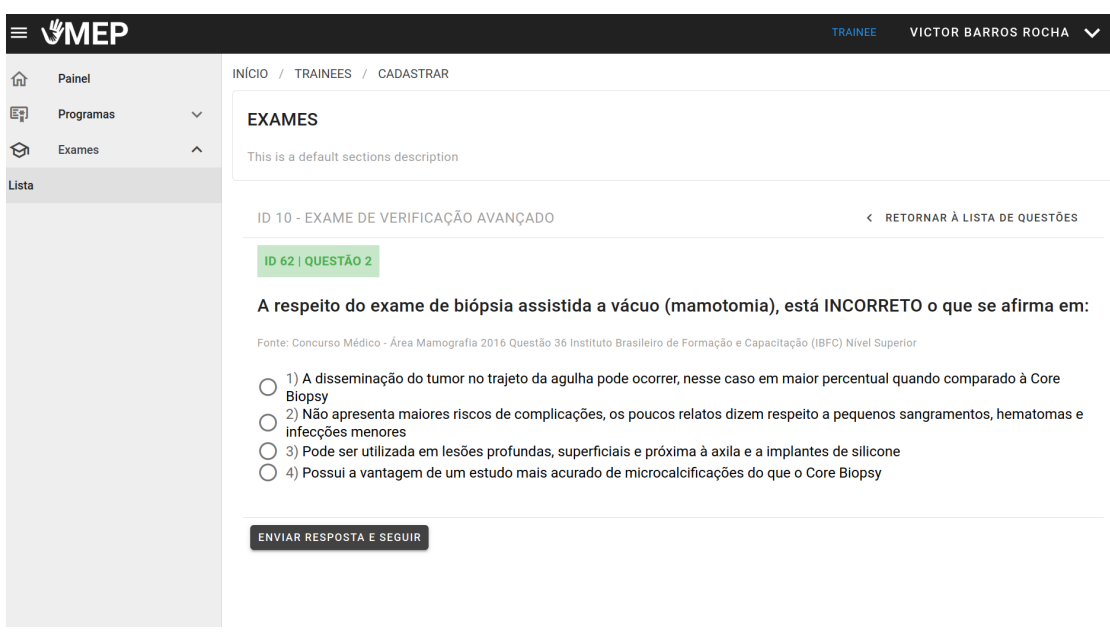

Figura 8. Captura de tela exibindo uma questão de um exame.

O módulo de questionários e gerenciamento também conta um banco de questões, nos quais os supervisores podem adicionar novos temas e criar exames específicos para cada trainee. Os temas podem abordar anatomia, operação de equipamentos, posicionamento, entre outros. Assim, um programa pode ser customizado para abordar falhas técnicas específicas de um ou mais trainees.

Os supervisores podem acompanhar o andamento dos questionários respondidos, bem como as fases concluídas no módulo gamificado pelo trainee. Um relatório contendo estatísticas como taxa de acerto, tempo de resposta e tempo de conclusão das fases no módulo gamificado pode ser gerado pelo supervisor responsável na página do programa de treinamento atribuído.

\subsection{Integração dos Módulos}

Em um programa de treinamento, a conclusão por parte do trainee do módulo de gamificação é opcional, conforme as especificações deste programa. O supervisor é responsável por definir quais exames e etapas um usuário deverá concluir para ser aprovado no programa. 
Ao iniciar um programa de treinamento, um arquivo de integração em formato JSON é gerado, caso o supervisor responsável tenha atribuído a tarefa de passar pelo módulo de gamificação. Este arquivo deve ser carregado pelo trainee ao iniciar a execução do módulo de gamificação. Desta forma, o trainee trabalha como um agente integrador dos módulos.

O trainee deverá então, realizar as etapas do módulo de gamificação (caso estabelecido) e responder os questionários atribuídos. Após a finalização do módulo de gamificação, o trainee é responsável por submeter os dados gerados durante a execução das fases. Esses dados são armazenados em banco de dados e processados para geração do relatório de desempenho. A ordem de execução dos módulos não é relevante.

\section{Discussão}

A falta de treinamento contínuo e defasagem no conteúdo de cursos de radiologia é um problema a ser abordado no Brasil. Países com renda média e baixa carecem de estrutura para treinar e educar os profissionais na área de radiologia [Sabino et al. 2014]. Em países desenvolvidos, o tempo de formação destes profissionais é de 48 meses, enquanto no Brasil leva-se 18 meses [Sabino et al. 2014].

Ainda, problemas como a falta de treinamento contínuo em centros de mamografia isolados, contribuem para uma baixa qualidade nas imagens mamográficas, resultando em falsos diagnósticos devido a falhas técnicas. Desta forma, este trabalho propôs um sistema educacional aberto voltado para a mamografia, como recurso mitigador deste cenário, visando a utilização em cursos técnicos, cursos de graduação e centros de treinamento, presencial e à distância.

O sistema construído também fornece insumos para avaliação do desempenho de profissionais de radiologia a partir de questionários. Assim, acredita-se que o sistema tem potencial para ser utilizado em programas de controle de qualidade e reciclagem.

No módulo do jogo, a partir das técnicas de gamificação como evolução em níveis (fases) e sistema de recompensa, espera-se que os usuários tenham um maior nível de engajamento e satisfação de uso enquanto aprende a operar o equipamento.

\section{Conclusão}

O sistema apresentado segue uma tendência na utilização de jogos para treinamento em diversas áreas [Dörner et al. 2016]. A possibilidade de extensão do módulo gamificado apresentado, bem como a possibilidade de customização dos programas de treinamento, permite que estudantes e técnicos de radiologia tenham treinamento customizado e específico de acordo com suas necessidades e experiência.

Como trabalho futuro, pretende-se avaliar o sistema com supervisores, estudantes e técnicos de radiologia. Pretende-se, também, incorporar ao módulo gamificado elementos mais avançados, como a possibilidade de compressão da mama. Atualmente técnicas de manipulação de elementos complexos (corpos macios - soft body physics) demandam um alto processamento dos computadores, não sendo amplamente aplicada em jogos.

\section{Agradecimentos}

Os autores agradecem à CAPES (Coordenação de Aperfeiçoamento de Pessoal de Nível Superior) pelo auxílio financeiro dado a esta pesquisa. 


\section{Referências}

Agius, E. C. and Naylor, S. (2018). Breast compression techniques in screening mammography-a maltese evaluation project. Radiography, 24(4):309-314.

Bigdeli, S. and Kaufman, D. (2017). Digital games in medical education: Key terms, concepts, and definitions. Medical journal of the Islamic Republic of Iran, 31:52.

Bray, F., Ferlay, J., Soerjomataram, I., Siegel, R. L., Torre, L. A., and Jemal, A. (2018). Global cancer statistics 2018: Globocan estimates of incidence and mortality worldwide for 36 cancers in 185 countries. CA: a cancer journal for clinicians, 68(6):394-424.

Bridge, P., Gunn, T., Kastanis, L., Pack, D., Rowntree, P., Starkey, D., Mahoney, G., Berry, C., Braithwaite, V., and Wilson-Stewart, K. (2014). The development and evaluation of a medical imaging training immersive environment. Journal of medical radiation sciences, 61(3):159-165.

Coop, P., Cowling, C., and Lawson, C. (2016). Tomosynthesis as a screening tool for breast cancer: A systematic review. Radiography, 22(3):e190-e195.

Cyrino, G., Tannús, J., Lamounier, E., Cardoso, A., and Soares, A. (2018). Serious game with virtual reality for upper limb rehabilitation after stroke. In 2018 20th Symposium on Virtual and Augmented Reality (SVR), pages 238-242. IEEE.

De Gloria, A., Bellotti, F., and Berta, R. (2014). Serious games for education and training. International Journal of Serious Games, 1(1).

Dörner, R., Göbel, S., Effelsberg, W., and Wiemeyer, J., editors (2016). Serious Games Foundations, Concepts and Practice. Springer International Publishing.

INCA - Instituto Nacional de Câncer José Alencar Gomes da Silva (2020). Estimate/2020 - cancer incidence in brazil.

Malhotra, V. M., Kabra, P. R., and Malhotra, R. (2017). Attitudes and practices of medical students regarding video-games: should community medicine educationists get serious about serious games. International Journal of Community Medicine and Public Health, 4(3):729.

O’Connor, M., Stowe, J., Potocnik, J., Giannotti, N., Murphy, S., and Rainford, L. (2021). $3 \mathrm{D}$ virtual reality simulation in radiography education: The students' experience. $R a$ diography, 27(1):208-214.

Sabino, S. M. P. D. S., Silva, T. B., Watanabe, A. H. U., Syrjänen, K., Carvalho, A. L., and Mauad, E. C. (2014). Implementation of a clinical quality control program in a mammography screening service of brazil. Anticancer research, 34(9):5057-5065.

Sapkaroski, D., Baird, M., McInerney, J., and Dimmock, M. R. (2018). The implementation of a haptic feedback virtual reality simulation clinic with dynamic patient interaction and communication for medical imaging students. Journal of Medical Radiation Sciences, 65(3):218-225.

Wang, R., DeMaria Jr, S., Goldberg, A., and Katz, D. (2016). A systematic review of serious games in training health care professionals. Simulation in Healthcare, 11(1):4151. 